\title{
HIGH LEVELS OF GENE FLOW IN THE CALIFORNIA VOLE (MICROTUS CALIFORNICUS) ARE CONSISTENT ACROSS SPATIAL SCALES
}

\author{
Rachel I. Adams ${ }^{1,2}$ and Elizabeth A. Hadly ${ }^{1}$
}

\begin{abstract}
Gene flow links the genetic and demographic structures of species. Despite the fact that similar genetic and demographic patterns shape both local population structure and regional phylogeography, the 2 levels of population connectivity are rarely studied simultaneously. Here, we studied gene flow in the California vole (Microtus californicus), a small-bodied rodent with limited vagility but high local abundance. Within a $4.86-\mathrm{km}^{2}$ preserve in central California, genetic diversity in 6 microsatellites was high, and Bayesian methods indicated a single genetic cluster. However, individual-based genetic analysis detected a clear signal for isolation-by-distance (IBD) and fine-scale population structure. Mitochondrial cytochrome $b$ sequencing revealed 11 unique haplotypes from the one local area where we sequenced 62 individuals. Phylogeographic analysis of these individuals combined with those sampled from the northern geographic range of the species (the range of the species spans western North America from southern Oregon to northern Mexico and is centered geographically within the state of California) again indicated a lack of structure but a signal for IBD. Patterns of gene flow thus are consistent across spatial scales: while dispersal of the California vole is limited across geographic distance, there is nonetheless considerable movement across the landscape. We conclude that in this species, high local population abundances overcome the potential genetic and demographic effects of limited dispersal.
\end{abstract}

Key words: fine-scale genetic structure, population genetics, phylogeography, Jasper Ridge Biological Preserve, cytochrome b, Bayesian analysis, SIMCOAL.

The geographic distribution of genetic diversity in natural populations harbors a wealth of information on species' evolutionary and ecological histories, demographic parameters, and conservation statuses. Gene flow, mediated by the movement of reproductive individuals and therefore genes across space, is a prominent determinant of the genetic composition and diversity within a species and ultimately of the species' survival. The role of gene flow in shaping the pattern of gene frequencies depends on ecological, historical, and demographic factors. Therefore, measurements of gene flow give insight into the behavior, migration, and mating patterns of natural populations. Gene flow is traditionally considered at 2 different temporal scales: for short distances and times, gene flow is a process, along with genetic drift, mutation, and natural selection, that shapes population genetic structure (Hartl and Clark 1997); at longer distances and times, gene flow, and the lack thereof, determine a species' phylogeographic pattern.

While both local population structure and phylogeography ultimately study the same evolutionary process-that of movement of individuals across space and time- - the demographic characteristics at the different scales vary, and consequently the patterns may be quite distinct. Nevertheless, the 2 levels of gene flow are rarely studied concurrently. For a species with limited vagility and strong philopatry but high abundance, expectations for signals of gene flow may differ depending on spatial scale considered. A strong signal of genetic structure is expected on both a microgeographic and macrogeographic scale for organisms with limited vagility, high fidelity to natal site, and specific habitat requirements (Lowe et al. 2004). However, species with high abundances have the potential to override factors limiting gene flow through sheer force of numbers, and therefore, genetic differentiation on a microgeographic scale, but not necessarily on a macrogeographic scale, would be lacking in these species. We set out to test whether signals of gene flow would be similar at different spatial scales for a species characterized by low dispersal, high philopatry, and large population sizes. By using 2 genetic markers of variable mutation rates (microsatellites and cytochrome $b$ ) from the same Microtus californicus individuals 
in one locality and linking these data to a study of the phylogeography of the species that used cytochrome $b$ (Conroy and Neuwald 2008), we were able to examine genetic structure across a small scale and place the locality into the phylogeographic structure of the species-effectively synthesizing patterns of gene flow at 2 spatial scales.

The California vole (Microtus californicus) is a small rodent $(<100 \mathrm{~g})$ whose demographic and ecological features have been well characterized. Individual dispersal distances are estimated to be small, at $50 \mathrm{~m}$ or less (e.g., Lidicker 1973, Bowen 1982), and philopatry high, with male home ranges slightly larger than female home ranges (e.g., $180 \mathrm{~m}^{2}$ and $120 \mathrm{~m}^{2}$, respectively; Heske 1987). California voles, like many other microtine rodents, undergo 2- to 4-year population cycles (Krebs 1966). In addition, the California vole is limited to areas of heavy ground cover, predominantly grasslands and oak woodlands, where it lives in underground burrows but travels aboveground to forage for grasses, herbs, and sedges. It breeds throughout the year, although reproduction peaks whenever food and cover are most abundant. Litter size averages 4 individuals but ranges from 1 to 9 , and females produce 2-5 litters per year. Females reach sexual maturity at an average of 29 days. The California vole's circadian activity, along with its widespread and abundant distribution, make it an important prey for carnivores. Although it has a broad range, from Baja California throughout western and central California to southern Oregon, the vole's populations are naturally fragmented into suitable habitat, which is patchily distributed across this range.

The particular biology of the California vole and other voles leads to the presence of population structure on a small spatial scale due to habitat patchiness and landscape features (Neuwald 2010, Berthier et al. 2005, Gauffre et al. 2008), as well as demography (Bowen 1982, Berthier et al. 2006, Gauffre et al. 2008). In southern California, a subspecies of the California vole which inhabits distinct marshes adjoining the Amargosa River in the Mojave Desert, M. c. scirpensis, showed reduced genetic diversity and a greater degree of subdivision between subpopulations than a more widely distributed subspecies, M. c. sanctidiegi (Neuwald 2010). Moreover, dispersal distances can vary depending on population density, season, and microhabitat (Krebs 1966, Lidicker 1973,
Heske 1987, Salvioni and Lidicker 1995), and this variation is known to affect the genetic diversity and structure of populations. Using allozyme data from M. californicus, Bowen (1982) found significant genetic differentiation between populations separated by only $50-200$ $m$ when population density was low. This differential disappeared when density rebounded. A similar recovery of connectivity during periods of high density has been observed in another vole, Arvicola terrestris (Berthier et al. 2006). However, other vole species show increased gene flow during periods of low density (Andreassen and Ims 2001, Hadly et al. 2004).

On a larger spatial scale, Microtus californicus has 17 distinct subspecies (Hall 1981) that span the range of the species and are based on pelage, skull shape, and body size (Kellogg 1918). However, further work based on distinct morphological differences, decreased fertility in crosses, and allozyme variation has shown a break between only 2 broad regions-northern and southern California (Gill 1980). Recently, a phylogeographic study of the California vole corroborated this geographic barrier, reporting strong genetic differentiation between populations on the northern and southern sides of the Transverse Mountain range in southern California (Conroy and Neuwald 2008).

Thus, previous work with California voles indicates that barriers to gene flow occur on temporal scales as well as local and regional spatial scales, but never before have the same individuals been used to determine gene flow at the 2 spatial scales simultaneously. For this goal, our study sampled heavily in one locality, the Jasper Ridge Biological Preserve (JRBP), San Mateo County, located in the San Francisco Bay region of California. The preserve itself represents habitat within an increasingly developed area, and JRBP is varied in particular landscape features. The preserve contains both native and nonnative grasslands. Nonnative grasses, predominantly of Mediterranean origin, dominate the nonserpentine grasslands, which are located on greenstone, sandstone, and chert substrates. And native grasses, which are adapted to the low nutrient content and the unique chemical content of serpentine grasslands, remain dominant on the serpentine-substrate outcroppings (Hobbs et al. 1997 and citations therein). It is not known whether small mammals have responded to this shift and partitioning of grassland types. Moreover, the 


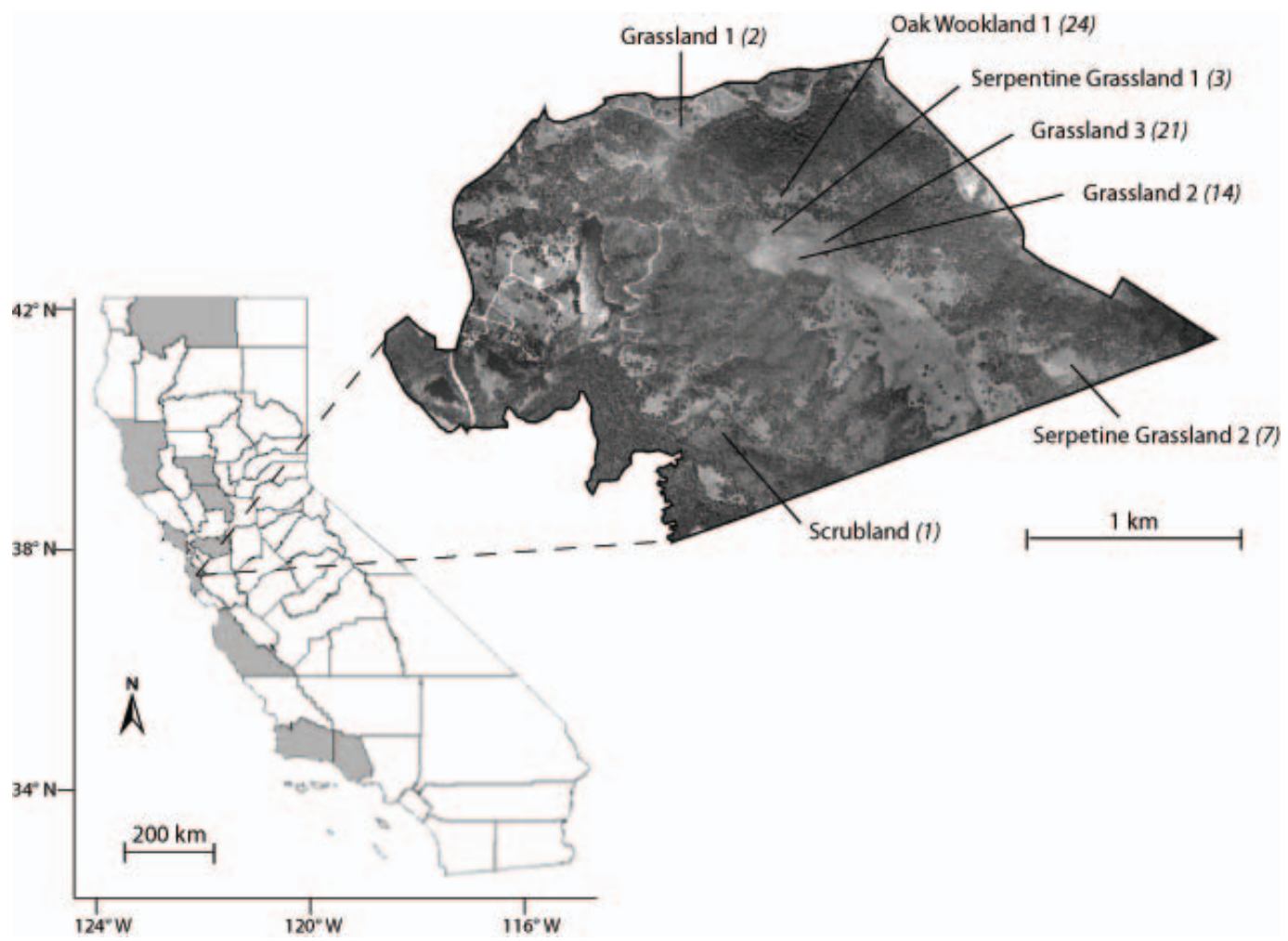

Fig. 1. Jasper Ridge Biological Preserve, San Mateo County, showing its location in the San Francisco Bay area of central California. Microtus californicus was trapped in 7 sites representing different habitat types in the preserve. Sampling numbers at each site are given in parentheses. Individuals from the highlighted California counties were included in the phylogeographic analysis.

preserve is varied in habitat, where areas of grasslands are of variable sizes and are separated by other, less preferred habitat types, such as those dominated by dense woody and/or shrubby vegetation.

This study examined the genetic signature of neutral microsatellite loci and examined the connectivity of California voles within a protected preserve characterized by distinct juxtaposed landscape features, including compositional shifts in grassland types separated by unsuitable forested habitat. We also used mitochondrial DNA to determine how these same JRBP California vole individuals were phylogenetically related to other populations across the species' range.

\section{Methods}

\section{Study Site and Animal Collection}

Tissue sampling was performed as part of a larger trapping study aimed at detecting the diversity and abundance of small mammal populations at JRBP, a $4.86-\mathrm{km}^{2}$ preserve in the foothills of the Santa Cruz Mountains managed by Stanford University (Fig. 1). Briefly, live Sherman and Tomahawk traps were laid out in 50-m-radius circular arrays or 100-m line transects in different sites representing the diversity of habitats at the preserve. Trapping arrays in different habitats varied in separation from 0.28 to $2.11 \mathrm{~km}$ with a median of $0.75 \mathrm{~km}$. Individual traps were baited with a mixture of oats and peanut butter and were opened for 5 consecutive nights during March and April 2006. All trapped animals were scored for species identity, sex, reproductive condition, and weight. Tissue samples (initially toe clips, later ear snips) were collected and stored in $100 \%$ ethanol in a refrigerator until DNA was extracted. The subspecies distinction for the site of JRBP is M. c. californicus (Hall 1981), and all individuals trapped were classified as belonging this subspecies. Trapping methods 
followed the American Society of Mammalogists guidelines and were approved by the Institutional Animal Care and Use Committee at Stanford University.

\section{Genetic Data Collection}

All 72 M. californicus tissue samples collected during the trapping study were included in the analysis. Animal material was dissociated using tweezers prior to extraction, and genomic DNA extraction followed the Qiagen (Valencia, CA) DNeasy protocol.

\section{Microsatellites}

A total of 6 nuclear microsatellite loci were used. Neuwald (2010) optimized PCR conditions for 5 primers (MSMM-2, 3, 5, 6, and 8) originally developed for Microtus montebelli (Ishibashi et al. 1999); an additional polymorphic locus (Moe2) was adapted from primers originally developed for Microtus oeconomus (Van de Zande et al. 2000). All microsatellite loci contain a dinucleotide repeat sequence. Tagged fluorescent primers were synthesized by Applied Biosystems (Foster City, CA) to allow for multiplexing: MSMM-2 and 8 were tagged with 6Fam (blue), MSMM-3 and 6 with VIC (green), and MSMM-5 and Moe2 with NED (yellow). Each PCR was carried out in a total volume of $10 \mu \mathrm{L}$ containing up to $5 \mu \mathrm{L}$ of DNA template, $2.5 \mu \mathrm{M}$ of each primer, $2.5 \mathrm{mM}$ dNTPs, $1.5 \mathrm{mM}$ $\mathrm{MgCl}_{2}, 5 \mathrm{U}$ of Taq DNA polymerase, and $1 \mu \mathrm{L}$ 10X PCR buffer from Invitrogen (Carlsbad, CA). PCR cycling conditions were as follows: an initial denaturation at $93^{\circ} \mathrm{C}$ for 2 minutes followed by 35 cycles consisting of 30 seconds at $90^{\circ} \mathrm{C}$, 20 seconds at $50-52{ }^{\circ} \mathrm{C}, 20$ seconds at $72{ }^{\circ} \mathrm{C}$, and a final 3 minutes of annealing at $72^{\circ} \mathrm{C}$. Samples were prepared with $\mathrm{Hi}-\mathrm{Di}^{\mathrm{rм}}$ formamide and $500 \mathrm{LIZ}^{\circledR}$ size standard and run on the 3730xl DNA Analyzer (Applied Biosystems, Foster City, CA) at the University of California, San Francisco, Genome Core Facilities.

\section{Mitochondrial Cytochrome $b$}

The primers MVZ 05 (Smith and Patton 1993) and vole-14 (Hadly et al. 2004) were used to amplify the entire cytochrome $b$ mitochondrial region (1143 bps). PCR was carried out in a total volume of $10 \mu \mathrm{L}$ containing $2 \mu \mathrm{L}$ of DNA template, $5 \mu \mathrm{M}$ of each primer, $2.5 \mathrm{mM}$ dNTPs, $1.5 \mathrm{mM} \mathrm{MgCl} 2,5 \mathrm{U}$ of Taq DNA polymerase, and $1 \mu \mathrm{L} 10 \mathrm{X}$ PCR buffer. PCR cycling conditions were as follows: an initial denaturation at
$94{ }^{\circ} \mathrm{C}$ for 1 minute followed by 40 cycles consisting of 15 seconds at $94{ }^{\circ} \mathrm{C}, 15$ seconds at 45 ${ }^{\circ} \mathrm{C}$, 45 seconds at $72{ }^{\circ} \mathrm{C}$, and a final 3 minutes of annealing at $72{ }^{\circ} \mathrm{C}$. Products were sequenced using the 3730 DNA Analyzer (Applied Biosystems) at Cogenics, Inc. (Houston, TX). Alignment was straightforward and done with Sequencher 4.7 (GeneCodes), and unique sequences have been deposited into GenBank, accession numbers GQ168680-GQ168690. Complete cytochrome $b$ sequences from individuals belonging to the northern clade of California voles (Conroy and Neuwald 2008) were retrieved from GenBank and used in phylogeographic analyses.

\section{Genetic Analysis}

Microsatellite Diversity.-The sampling protocol designed to survey small mammals in general, rather than $M$. californicus in particular, led to highly variable sample sizes for each predefined trapping locality, with many of the localities containing only a few individuals (Fig. 1). Therefore, individual-based, rather than population-based estimates of genetic structure were used for all microsatellite analyses. Population-based analyses require the assignment of individuals to predefined populations; using individual organisms as the unit of analysis has the advantage of utilizing all available genetic information and requires fewer a priori assumptions. Summary statistics and basic measures of genetic diversity were generated in GenAlEx (Peakall and Smouse 2006). GenePop (Rousset 2008) was used to test for deviations from Hardy-Weinberg equilibrium using an exact test and also for evidence of linkage disequilibrium using the $G$ test.

Detecting Genetic STRUCTURE.-At JRBP, several characteristics independent of distance have the potential to limit vole dispersal. First, several of the grasslands where the California voles were collected are separated by unsuitable habitat for this species, such as forest and dense chaparral. Second, the grasslands are represented by 2 types - serpentine and nonserpentine-and it was unknown whether the voles discriminated between these 2 types. Therefore, we sought to determine if there was any population subdivision within JRBP. We used 2 Bayesian-based programs, the spatially nonexplicit Structure 2.2 (Pritchard et al. 2000) and the spatially explicit Geneland (Guillot et al. 2005, 2008), to cluster individuals into 
predefined $K$ populations that minimize linkage disequilibrium (LD) and maximize HardyWeinberg equilibrium. Within Structure, 10 independent runs for a fixed $K=1$ to 7 were carried out, with a maximum of 7 defined by the number of trapping arrays. We assumed admixture and a model of correlated allele frequencies between populations (Falush et al. 2003) with a burn-in period of 100,000 iterations followed by 1,000,000 Markov Chain Monte Carlo (MCMC) replicates. The assumption of correlated allele frequencies better handles departures from Hardy-Weinberg equilibrium or LD; however, it can also lead to overestimating $K$ (Pritchard et al. 2007), so particular attention was paid to this possibility during analysis. Time estimates were determined by ensuring that parameter estimates converged before the end of the burn-in period, and the method developed by Evanno et al. (2005) was employed to calculate the most likely value of $K$. Geneland incorporates the geographic location of sampling in estimates of population structure. $K$ was allowed to vary in 10 independent runs, and the most probable value was estimated using 1,000,000 MCMC iterations, with the maximum rate of the Poisson process fixed at 500, the maximum number of nuclei in the Poisson-Voronoi tessellation fixed at 200, and use of the Dirichlet model for allele frequencies.

SCALES OF GENETIC STRUCTURE.-We sought to identify the geographic scale of genetic structure at JRBP independent of delimited populations, and for this we employed a number of analyses in GenAlEx. Principle coordinates analysis (PCA) was used to plot the relationships among all individuals. In order to test whether individuals collected from within serpentine grasslands were more genetically similar than individuals from across different grassland types, we performed a Mantel test with genetic distance and serpentine/nonserpentine distinction. Isolation-by-distance (IBD) was tested in several ways. To test the statistical relationship between geographic distance and the genetic distance matrices, we performed a Mantel test, where significance was determined compared to 1000 permutations. Moreover, in order to test for fine-scale genetic structure, we employed a heterogeneity test for autocorrelation of pairs of individuals within defined distance classes (Smouse et al. 2008). A distance class of $400 \mathrm{~m}$ was chosen based on estimates for home ranges of M. californicus measured at JRBP (Giusti
1985) and tested for significance with 1000 bootstraps. Similarly, Ritland's estimator of relatedness (Ritland 1996) was calculated for each individual pairwise comparison, and this estimate was correlated with geographic distance using Pearson's product moment correlation. We further sought to estimate the dispersal distance of voles in this population, and to this end we utilized the relationship between pairwise genetic distance and neighborhood size developed by Rousset (2000). Dispersal distance can be estimated according to $\sigma^{2}=1 /(4 D \pi b)$, where $\sigma$ is the mean parent-offspring dispersal distance, $D$ is the effective density of the population, and $b$ is the slope of the regression of pairwise genetic distance (Rousset's $a$ ) against the logarithm of geographic distance. Rousset's $a$ was generated with the program SPAGeDi (Hardy and Vekemans 2002), and 2 values for $D$ were used due to the cyclical nature of vole populations, one during high animal density $\left(0.101\right.$ voles $\left.\cdot \mathrm{m}^{-2}\right)$ and one during low animal density $\left(0.017\right.$ voles $\left.\cdot \mathrm{m}^{-2}\right)$. These estimates of density were informed by a field study previously conducted at JRBP (Giusti 1985). Although the approximate linear relationship between genetic and geographic distances is not expected to hold as well at distances less than the estimated demographic dispersal distance (Rousset 1997), we did not exclude any pairwise comparisons because the geographic center of the trapping array was used as the trapping point rather than the specific location of the trap. Regardless, the slopes of the linear regression have been shown to differ little whether pairwise comparisons less than the demographic dispersal distance are excluded or not (Rousset 2000).

To look for evidence of a recent reduction in effective population size, we used the program Bottleneck v.1.2.02 (Piry et al. 1999). This program tests for a severe population reduction using the principle that populations that have undergone a bottleneck demonstrate a reduction in the number of alleles as well as in heterozygosity (sensu Nei 1987), with decrease in allele number occurring faster than loss of heterozygosity. Under this expectation, the observed heterozygosity is higher than the expected heterozygosity from the allele number at mutationdrift equilibrium (Cornuet and Luikart 1996). Within Bottleneck, we implemented a Wilcoxon test assuming the 2-phase model (TPM) of microsatellite evolution with $95 \%$ of mutations 
following a single-step model and 5\% a multistep model.

We compared the results of the microsatellite analyses with those reported in another study examining these same loci in other populations of $M$. californicus (Neuwald 2010). While we cannot compare absolute sizes of microsatellites because we did not standardize across genotyping runs and because of limited gene flow between northern and southern clades of this species (Conroy and Neuwald 2008), we are nevertheless able to compare relative numbers and range of alleles across the 2 studies.

CYTOCHROME B AND PHYLOGEOGRAPHY.-We looked for evidence of nuclear mitochondrial DNA inserts (Numts) by examining patterns of substitution. We checked for the presence of premature stop codons and a bias in substitutions in the 3rd codon position relative to the 1st and 2nd positions, which tend to be conserved because changes in these sites often result in amino acid replacements. EstimateS (Colwell 2006) was used to generate a haplotype accumulation curve, where the expected richness function, Mao Tau, and the standard deviation were calculated using the analytical formulas of Colwell et al. (2004) and scaled against the computed individuals in the sampling effort (Gotelli and Colwell 2001).

In order to incorporate individuals from JRBP into the biogeographic history of the species, we employed standard phylogeographic techniques. Because Conroy and Neuwald (2008) found little evidence for gene flow between the northern and southern clades of $M$. californicus, and because we did not detect any sequences from the southern clade in this study, we used complete sequences $(n=19)$ from just the northern clade. Mantel tests using genetic distance between cytochrome $b$ haplotypes were conducted in GenAlEx at 2 spatial scales: JRBP individuals exclusively $(n=11)$ and JRBP individuals plus ones from across the northern California range $(n=30)$.

Maximum parsimony with 1000 bootstrap replicates was determined in PAUP* (Swofford 2003) to examine the evolutionary history of California voles. We limited our phylogenetic analysis to parsimony because of the reduced likelihood of homoplasy in our recently diverged samples. Exploration using maximum likelihood and Bayesian techniques produced similar results (results not shown). A representative from the southern clade (haplotype \#S13 from
Conroy and Neuwald [2008], GenBank accession \#EF506039) served as the outgroup, from which all northern samples were forced to be monophyletic. Parsimony analysis was performed using stepwise random addition sequence and TBR-branch swapping, and 1000 bootstrap replicates were executed. A haplotype network was generated using TCS version 1.21 (Clement et al. 2000) with a $95 \%$ confidence interval for assigning branches, excluding the outgroup, which required a lower threshold value. Representation of the haplotype network generated by TCS was subsequently generated by hand.

Because the high diversity in mitochondrial haplotypes from a single locality indicated that California voles were extremely abundant, Bayesian serial coalescent modeling was used to estimate the effective population size of the JRBP individuals. We used this technique to simulate the number of segregating sites and examine the probability of detecting the observed level of diversity (i.e., 11 segregating sites) under different mutation rates and effective population sizes. We explored the effect of deme size from 30,000 to 600,000 individuals, at 3 mutation rates, $\mu$, corresponding to low ( $2 \%$ per million years per base pair [bp]), moderate (4\%), and high (10\%) values (Hadly et al. 2004). We assumed 5 generations per year (Greenwald 1957), a constant growth rate, a uniform mutation rate, and a sequence length of $1143 \mathrm{bp}$. Running the model 10,000 times at each parameter combination provided the expected distribution of gene diversity. Bayesian Serial SimCoal (http://www.stanford.edu/group/hadly lab/ssc/BayeSSC.htm) is a modification of SIMCOAL 1.0 (Excoffier et al. 2000).

\section{RESUlts}

Seventy-two M. californicus individuals were collected from 7 trapping sites (median individuals captured $=7$ ) across JRBP in predominantly grassland habitats (Fig. 1). The only vole captured outside a grassland was a pregnant female, which was trapped in a woody scrubland; other woodland and forest trapping localities contained no voles. The scrubland in which the vole was captured is situated adjacent to small grassland pastures.

\section{Genetic Variation}

Genotype analyses revealed high levels of genetic diversity (Table 1). A total of 72 alleles 
TABLE 1 . Number of alleles $(\mathrm{A})$, expected heterozygosity $\left(H_{\mathrm{E}}\right)$, observed heterozygosity $\left(H_{\mathrm{O}}\right)$ averaged over individuals, and $P$ values for Hardy-Weinberg equilibrium (HWE; determined using chi-square exact tests) for Microtus californicus in this study (M. c. californicus) and for Neuwald (2010; M. c. scirpensis and M. c. sanctidiegi).

\begin{tabular}{|c|c|c|c|c|c|c|c|c|c|c|}
\hline \multirow[b]{2}{*}{ Locus } & \multicolumn{4}{|c|}{ M. c. californicus $(n=71)$} & \multicolumn{3}{|c|}{ M. c. scirpensis $(n=67)$} & \multicolumn{3}{|c|}{ M. c. sanctidiegi $(n=27)$} \\
\hline & A & $H_{\mathrm{E}}$ & $H_{\mathrm{O}}$ & $\begin{array}{c}\mathrm{HWE} \\
P \text { value }\end{array}$ & A & $H_{\mathrm{O}}$ & $\begin{array}{c}\mathrm{HWE} \\
P \text { value }\end{array}$ & A & $H_{\mathrm{O}}$ & $\begin{array}{c}\text { HWE } \\
P \text { value }\end{array}$ \\
\hline MSMM2 & 16 & 0.873 & 0.914 & 0.359 & 6 & 0.716 & 0.000 & 15 & 0.800 & 0.100 \\
\hline MSMM3 & 12 & 0.825 & 0.725 & 0.225 & 3 & 0.075 & 0.314 & 12 & 0.667 & 0.011 \\
\hline MSMM5 & 12 & 0.877 & 0.549 & 0.000 & 1 & 0 & - & 13 & 0.704 & 0.071 \\
\hline MSMM6 & 5 & 0.305 & 0.322 & 1.000 & 1 & 0 & - & 7 & 0.593 & 0.076 \\
\hline MSMM8 & 13 & 0.888 & 0.424 & 0.000 & 2 & 0.284 & 0.000 & 12 & 0.654 & 0.000 \\
\hline Moe2 & 14 & 0.881 & 0.871 & 0.001 & - & - & - & - & - & - \\
\hline
\end{tabular}

TABLE 2. Outcome of 2 individual-based Bavesian clustering programs. In Structure, 10 independent runs of $K$ fixed from 1 to 7 were completed, and the means and standard deviations of the log likelihood values are reported here. In Geneland, 10 independent runs were completed, and the estimated $K$ and $\log$ likelihood values are reported.

\begin{tabular}{llllr}
\hline \multicolumn{3}{c}{ Structure } & & \multicolumn{2}{c}{ Geneland } \\
\cline { 1 - 2 } \cline { 5 - 5 }$K$ & Log likelihood & & $K$ & Log likelihood \\
\hline 1 & $-1669.29 \pm 0.34$ & & 1 & -1219.6157 \\
2 & $-2029.33 \pm 118.79$ & & -1222.2049 \\
3 & $-1800.14 \pm 46.79$ & & -1196.9087 \\
4 & $-1892.94 \pm 67.72$ & & 1 & -1212.0824 \\
5 & $-1986.00 \pm 135.67$ & & & -1215.2921 \\
6 & $-1982.31 \pm 38.84$ & & 1 & -1227.3241 \\
7 & $-2017.74 \pm 45.13$ & & 1 & -1213.1726 \\
& & 1 & -1232.2259 \\
& & 1 & -1214.4837 \\
& & 1 & -1211.5334 \\
\hline
\end{tabular}

was found for the 6 microsatellite loci, with each locus supporting between 5 and 16 alleles, with an average of $12(\mathrm{SE}=3.7)$ alleles per locus. Expected heterozygosity for each locus ranged from 0.31 to 0.89 , with an average of 0.78 (SD $=0.23$ ) for all loci. Observed heterozygosity at each locus ranged from 0.32 to 0.91 , with an average of $0.63(\mathrm{SD}=0.24)$ for all loci. Three of the 6 loci (MSMM-5, MSMM-8, Moe2) demonstrated a heterozygosity deficit and significant deviation from Hardy-Weinberg equilibrium expectations (Table 1). Lower than expected levels of heterozygosity may be common in species experiencing large interannual cycles in population size (Delport et al. 2005). Three locus pairs, each including locus MSMM-5, showed significant linkage disequilibrium $(P<0.05)$. Analyses were explored without that locus, and because results were similar (data not shown), analyses include all loci. The level of sampling, both in terms of individuals caught and size of geographic area, matched that of the endangered subspecies M. c. scirpensis. However, the level of genetic diversity we found most closely mirrored the widespread subspecies $M$. c. sanctidiegi, which was assessed at a broader geographic scale-spanning 3 counties in southern California-but for fewer individuals (Neuwald 2010).

\section{Genetic Structure}

Clustering analysis performed in Structure and Geneland showed that Jasper Ridge represents a single genetic population (Table 2). In Structure, the estimated logarithm of likelihood was highest for $K=1$, and the method of Evanno et al. (2005) detected $K=1$ cluster. Individuals assigned to particular populations when $K>1$ were distributed randomly across trapping sites. Likewise, 10 independent runs of Geneland all showed the greatest likelihood at $K=1$. Furthermore, PCA analysis corroborated a lack of structure by demonstrating no obvious clustering of individuals by trapping locality or habitat type.

A Mantel test between pairwise geographic and genetic distances showed a significant result using microsatellites $(P=0.042)$. Similarly, there was a significant and positive relationship between Rousset's $a$ and geographic distance $(P=0.003)$. Using the slope of this regression $(b=0.00826)$ and the 2 estimates of density $(D)$, the indirect dispersal distance of voles $(\sigma)$ based on genetic data yields 2 values: $9.8 \mathrm{~m}$ at high vole density and $23.8 \mathrm{~m}$ at low vole density.

Spatial genetic autocorrelation analysis, including a heterogeneity test, showed evidence for microgeographic spatial genetic structure (Fig. 2). Correlations were near zero for the first distance classes, until the 1600-m distance interval, at which point correlations became highly negative. Interestingly, correlations were not positive at the short distance classes, as is often 


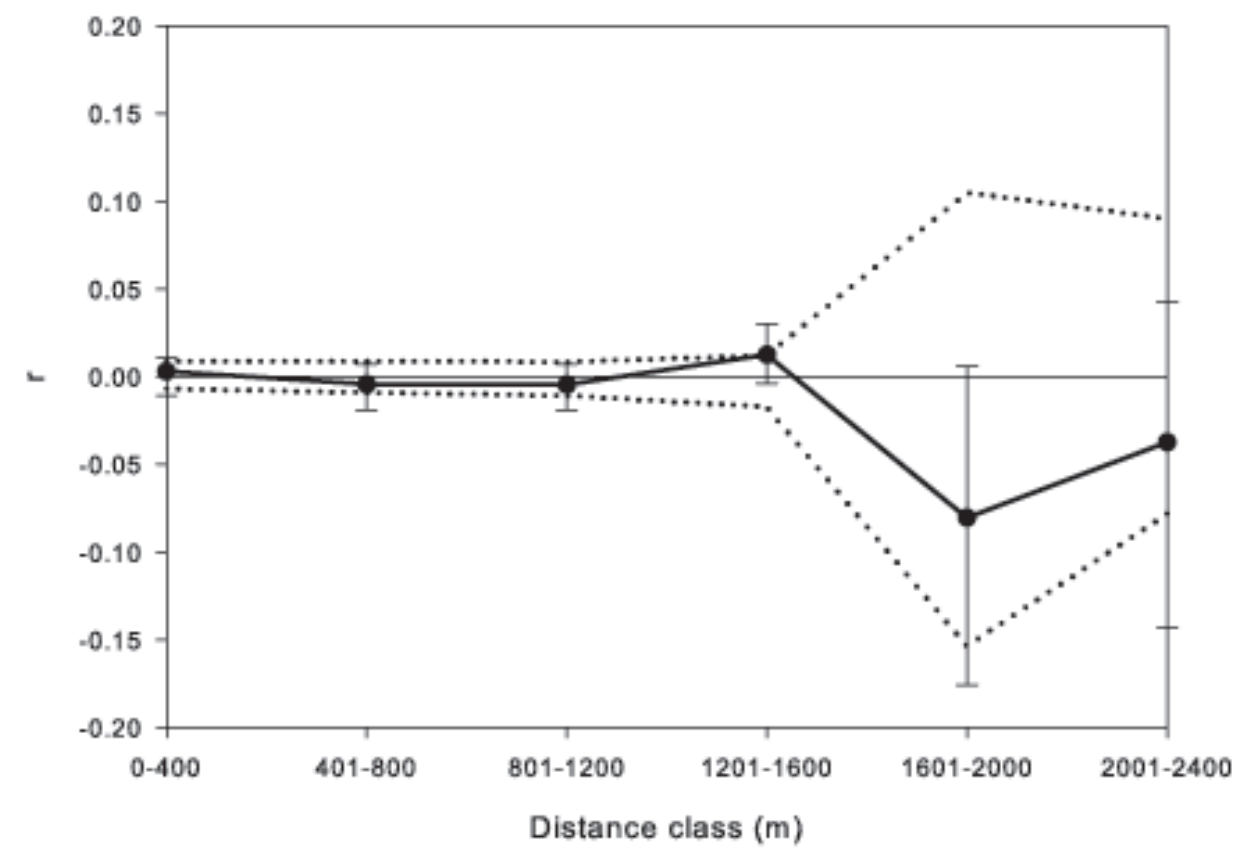

Fig. 2. Fine-scale correlogram showing the genetic autocorrelation $(r)$ as a function of distance partitioned into discrete size classes. Error bars bound the 95\% CI for $r$ determined with 1000 bootstrap trials for comparisons within size class; dashed lines show the 95\% CI for the null hypothesis determined by 1000 random permutations of individuals among geographic locations. Note the abrupt change in $r$ at distance classes $>1600 \mathrm{~m}$.

the case (Gauffre et al. 2008, Smouse et al. 2008). Although the overall correlogram was significant $(w=25.63, P=0.035)$, only one individual distance class was significant, and it was positive: $1200-1599 \mathrm{~m}$ (autocorrelation $r=0.013$, $P=0.036)$. However, the distance class 1600 $1999 \mathrm{~m}$ was marginally significant (autocorrelation $r=-0.080, P=0.065)$. The confidence interval for the values increases at the large distance classes due to a reduced number of comparisons in these ranges. Relatedness, as measured by Ritland's RI (Ritland 1996), was negatively correlated with geographic distance (correlation $r=-0.047, P=0.026$ ). There was no evidence for a population bottleneck (Wilcoxon, $P=0.578$ ), and a Mantel test showed a nonsignificant $(P=0.251)$ correlation between pairwise genetic distance and serpentine/nonserpentine sampling locality.

\section{Phylogeography and Cytochrome $b$}

None of the cytochrome $b$ genetic sequences had accumulated premature stop codons. The majority (73\%) of base pair (bp) changes were located in the 3rd position and did not result in an amino acid change (64\%). Therefore, haplotypes were treated as true mitochondrial sequence. Complete cytochrome $b$ sequences (1143 bp) were obtained from 62 individuals, and 11 distinct haplotypes were observed. $\mathrm{Nu}$ cleotide base composition was similar to that reported for other Microtus species: 29.0\% cytosine, $26.8 \%$ thymine, $32.3 \%$ adenine, and $12.0 \%$ guanine (Hadly et al. 2004, Spaeth et al. 2009). There were 11 total variable sites. Gene diversity $(\mathrm{Hd}=0.85 \pm 0.025)$ and nucleotide diversity $(\pi=0.0018 \pm 0.0001)$ were similar to those for other voles (Hadly et al. 2004, Spaeth et al. 2009).

Individual-based rarefaction analysis indicated that the sampling effort detected the preponderance of genetic diversity at JRBP (Fig. 3). The number of haplotypes detected with increased sampling effort had begun to saturate, as evidenced by a logarithmic line fit through the data points. This best-fit line predicts that sampling an additional 20 individuals would uncover 1 further haplotype. The locality of JRBP demonstrated a large amount of withinsite diversity, but detection of this haplotype diversity required intensive sampling. The entire set of 11 haplotypes appears in each of 


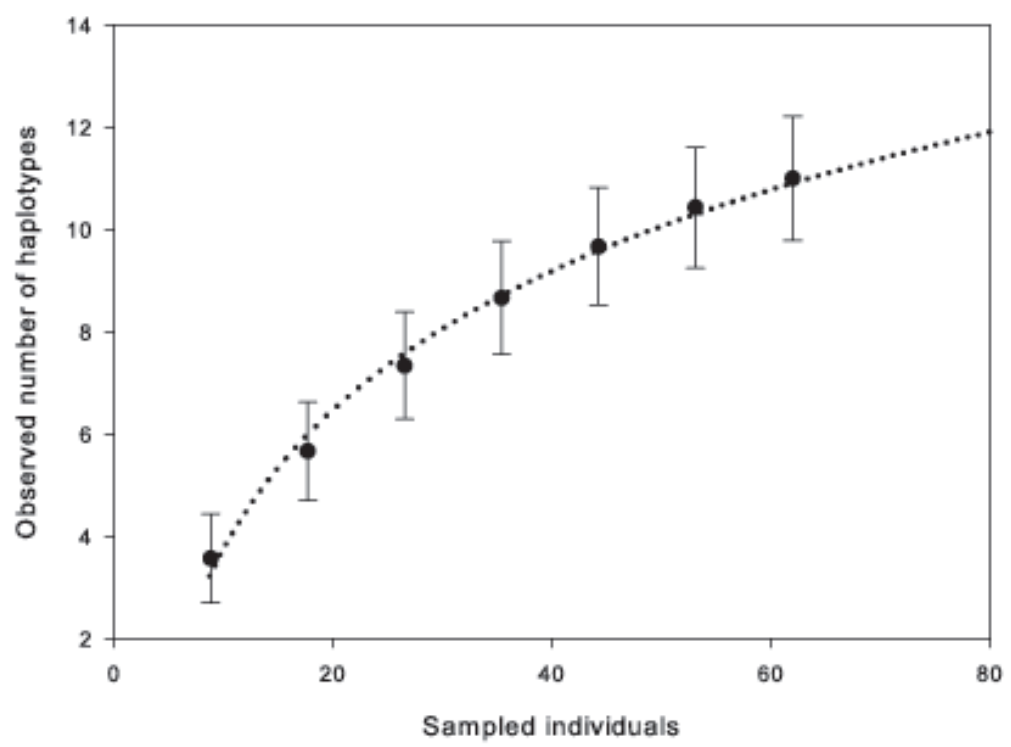

Fig. 3. Haplotype accumulation curve for mitochondrial cytochrome $b$ haplotypes at Jasper Ridge Biological Preserve. 95\% CI bars are shown. Included is a logarithmic line through the observed data points, showing the projected modest detection of haplotypes with increased sampling effort.

the 3 largest sampling localities, and the smaller sampling localities each contain a subset of these types. The most abundant haplotype $(n=$ 18) appeared in all sampling localities. All of the haplotypes detected at JRBP were undetected in the larger phylogeographic study of the species.

A Mantel test for IBD within the mitochondrial locus was not significant for those individuals within JRBP $(P=0.340)$ but was highly significant for all individuals across northern California $(P=0.001)$.

Phylogeographic analysis with maximum parsimony analysis produced 5 most-parsimonious trees. The $50 \%$ majority-rule consensus tree with bootstrap support is shown in Fig. 4. Microtus californicus individuals from JRBP fit into the northern clade, but there is little structure across the entire northern clade. Rather, some geographic areas show in situ diversification. In particular, haplotypes in Ventura County, Santa Barbara County, Jasper Ridge, Jasper Ridge/Yolo County, and Marin/Mendocino counties demonstrate diversification from a separate common ancestor. The haplotype network (Fig. 5) corroborates the limited presence of structure by geography. Southern lineages from within the northern clade (Ventura and Santa Barbara counties) are closely related, as are haplotypes from the northern central counties of Marin and Mendocino. There is also notable relatedness between the northern and southern extremes from the counties examined: haplotypes from Santa Barbara and Siskiyou counties.

Bayesian serial coalescent modeling indicated that the effective population size for M. californicus is large, at least 120,000 individuals (Fig. 6). Deme size at a low mutation rate peaked in likelihood at 400,000 individuals, at a moderate mutation rate at 210,000 individuals, and at a high mutation rate at 120,000 individuals.

\section{Discussion}

This study documents a high amount of gene flow in M. californicus, both on fine and coarse spatial scales. Microsatellites indicated that the JRBP, a $4.86-\mathrm{km}^{2}$ preserve, represents one breeding population with high diversity, nonetheless exhibiting IBD on a microgeographic scale of distances $<2 \mathrm{~km}$. At the same time, phylogeographic analysis revealed a complete lack of structure across the entire northern clade of the species, further indicating a high amount of movement and dispersal, while showing the pattern of IBD. Although nuclear microsatellites and mitochondrial sequence reveal gene flow on different timescales, both here show the same overall signal. 


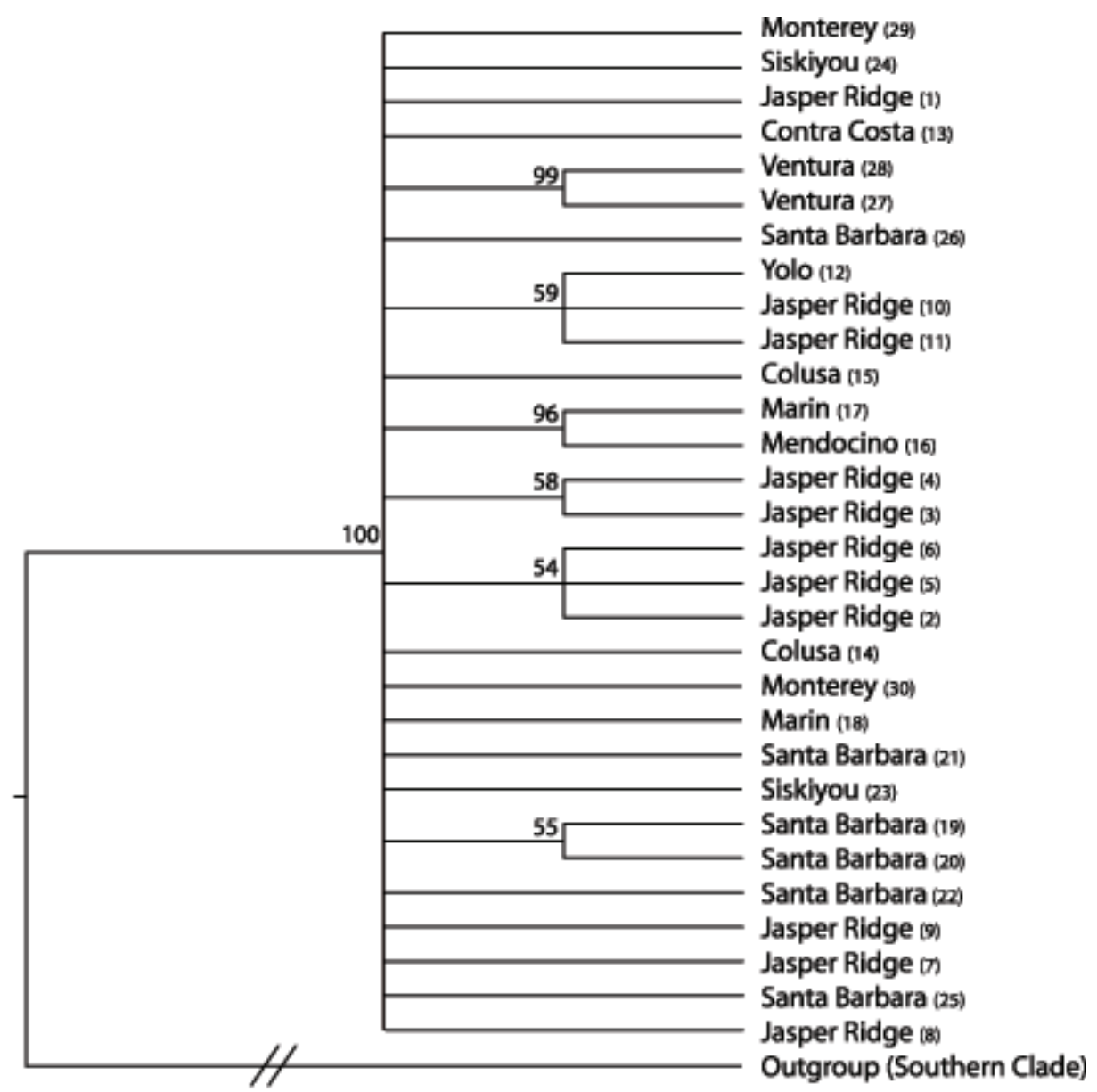

Fig. 4. Maximum parsimony tree with bootstrap support above the nodes generated from the complete mitochondrial cytochrome $b$ nucleotide sequence. Numbers in parentheses indicate the unique haplotype identity used in the haplotype network (see Fig. 5). Note the distribution of southern and northern variants throughout the tree.

The hypervariable microsatellites detected a signal of increasing difference with distance, but did not detect clear population structure. In this case, a significant signal for IBD did not lead to overestimation of genetic structure, as can be the case when Bayesian methods are employed (Frantz et al. 2009). A pattern of IBD without clearly limited subpopulations can be expected from a species whose distribution is continuous rather than discrete (Futuyma 1998). Therefore, the unfavorable habitat types-those containing woody and/or shrubby vegetation-within JRBP do not represent significant barriers to gene flow. Rather, from a genetic perspective, the voles represent a continuous population: despite limited dispersal and high philopatry, the movement and abundance of vole individuals appear great enough to prevent genetic differentiation.

Perhaps more suprisingly, the same pattern is observed across a much wider spatial scale within the entire northern clade of the species: phylogeographic analysis further showed that there is no geographic structure in individuals spanning southern to northern California. California vole movement across landscapes is high, such that the signal of IBD detected using nuclear microsatellite markers did not lead to a signal of phylogeographic structure using mitochondrial DNA. Despite the smaller effective population size of mitochondrial DNA relative to nuclear DNA, gene flow is sufficiently high on both a short- and long-term basis to prevent significant population structure. 




Fig. 5. Haplotype network for the complete mitochondrial cytochrome $b$ nucleotide sequence. Numbers identify unique, detected haplotypes, and small circles without numbers represent inferred haplotypes. Each line represents one mutational step, and additional mutational steps are represented by hatch marks. The size of the circle represents the frequency of haplotype detected, where the smallest circle $=1$ individual and the largest circle $=18$ individuals. Geographic location of samples is indicated with shading, where lighter circles are more southern (Ventura and Santa Barbara counties, haplotype \#19-22, 25-28) and darker circles are more northern (Siskiyou County, haplotype \#23-24). See Fig. 4 for the pairing of haplotype identity with precise sampling location. Haplotypes represent a panmictic population with some examples of in situ diversification within localities and of limited grouping by geographic location.

Gene Flow, Demography, and Landscape Features

Many species occur in subdivided populations due to spatial heterogeneity of suitable habitat. This fragmentation may be caused by a naturally patchy habitat, as can be the case when environmental variation leads to discontinuous distributions of ecosystems or habitat loss from anthropogenic disturbance. Our data supports a growing body of evidence that high levels of genetic diversity and connectivity in voles can be maintained in patchy habitats (Stewart et al. 1999, Aars et al. 2006). For example, the water vole Microtus richardsoni maintains high genetic diversity despite its restriction to isolated streamlets (Stewart et al. 1999). On the other hand, a subspecies of California voles residing in fragmented marshes demonstrates significant 


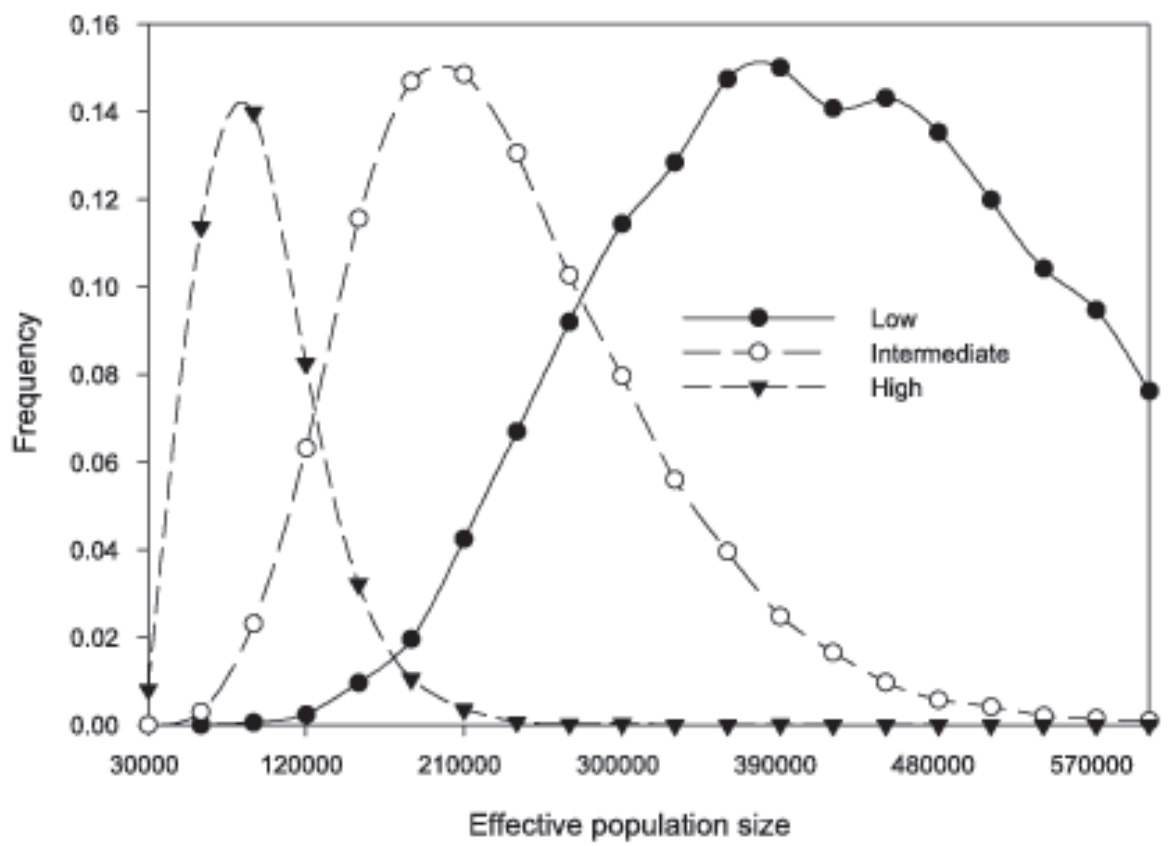

Fig. 6. Frequency of outcomes in Bayesian serial coalescent modeling that match the observed level of mitochondrial gene diversity. Results are shown for 3 mutation rates at a range of effective population sizes. For a high mutation rate, the effective population size most likely matching the observed diversity is 120,000 , while for a low mutation rate, the effective population size is approximately 400,000.

population structure (Neuwald 2010). Especially for species which occur in spatially discrete subpopulations, genetic structure can be complex (Mayer et al. 2009) and seems to vary depending on the population in consideration. Fragmentation can constrain gene flow, which serves to increase the rate of genetic drift and inbreeding (Frankham et al. 2002) over time. These processes decrease genetic diversity, which can have direct impacts on the fitness of a species (Soule 1980, Coltman et al. 1998) because genetic diversity and evolutionary potential of a species are correlated (Lavergne and Molofsky 2007). It is therefore important, from a conservation perspective, to describe the spatial population structure of natural populations, which can inform the extant level of gene flow, in order to predict how species may respond to further habitat fragmentation.

The maintenance of high genetic diversity at JRBP despite the spatial and demographic characteristics that would limit genetic diversity indicate that $M$. californicus has a large effective population size at this site. And in fact, findings from Bayesian serial coalescent modeling corroborated these findings. Such a large effective population size could prevent the development of both local and regional genetic structure as long as corridors of migration remain accessible. And in fact, the large deme size for individuals from JRBP indicates a high amount of genetic exchange with outside populations. A large effective population size as a factor overriding high philopatry in shaping genetic structure has been suggested in kangaroo rats (Dipodomys spp.; Waser and Elliott 1991). While the overall effective population size has likely remained high through time in M. californicus, local census populations are known to experience boom-and-bust cycles. Our JRBP study uncovers a snapshot of the local genetic structure, and without trapping over time, we are unable to determine the population abundance at the time of collection relative to the long-term average. However, the lack of population structure across geographic distances spanning $2 \mathrm{~km}$ supports the conclusion that our sampling spanned a period of high abundance (Bowen 1982).

Our estimates of gene flow are largely concordant with indirect dispersal distances based on mark-recapture demographic studies. 
Demographic studies have found dispersal distances to be quite small, predominantly less than $10 \mathrm{~m}$ (Lidicker 1973, Salvioni and Lidicker 1995), but a few individuals were caught at distances of $50 \mathrm{~m}$ from their home range (Bowen 1982). Other studies have shown genetic estimates to be in general concordance (Gauffre et al. 2008) to nearly half that of empirical estimates (Broquet et al. 2006). How dispersal distances differ depending on population sizes is an interesting component to this analysis. That voles may disperse different distances depending on the size of the population has been suggested for the California vole based on demographic sampling (e.g., Heske 1987), with the expectation that dispersal is a stochastic process with higher overall success during periods of high abundance that "wash out" potential subdivisions based on limited vagility and high philopatry (Bowen 1982). Here, however, genetic analysis from Rousset's $a$ indicates that a longer dispersal distance might be more common and successful during periods of low density, since dispersal distance was nearly 2.5 times lower during periods of high density (9.8 $\mathrm{m}$ at high density versus $23.8 \mathrm{~m}$ at low density). Other studies have found that a lower population size leads to a higher probability of successful distal gene flow (Andreassen and Ims 2001, Hadly et al. 2004). Dispersal may then mimic and be linked to the probability of reproductive success, which in voles can also be higher when density is lower (Krebs 1966). If California voles simultaneously demonstrate a pattern of high reproduction (Krebs 1966) and high dispersal (this study) during low population density, then this could result in sustained or even elevated genetic admixture during unfavorable conditions. Such a trait may prove highly beneficial to a species experiencing environmental change. In contrast to voles, for example, gophers do not disperse readily during years of low abundance, and therefore, environmental changes which reduce population size have a much higher chance of endangering local populations (Hadly et al. 2004).

\section{Phylogeography}

Over longer time scales, the San Francisco Bay has acted a barrier to dispersal, and the result of this barrier on the speciation and subspeciation of rodents has been studied previously (Hooper 1944). Microtus californicus, like many rodents of the area, demonstrated distinct morphological features in different areas around the bay. In the case of the California vole, subspeciation status was defined based on phenotypic differences of pelage, size of brain case/skull, size of rostrums and nasals, and overall size (length). Four subspecies were delimited in the San Francisco Bay Area. As is typical for other phylogeographic analyses based on neutral genetic differences, subspeciation distinctions based on phenotype were not detected in this study, indicating that the species is either phenotypically plastic or, more likely, prone to local adaptations or drifts of the phenotype. As recently as 18,000 years ago, however, the San Francisco Bay was a large continuous grassy valley (Atwater 1979), and the California vole would have been linked to neighboring regions. Therefore, the submerge that created the San Francisco Bay has led to phenotypic differences but has not resulted in phylogeographic isolation, as the individuals from JRBP on the San Francisco peninsula are part of a panmictic northern California population. Nevertheless, the lack of any overlap in haplotypes between the JRBP population and the larger geographic range, without any unique structure for the JRBP population within the northern range, indicates that California voles demonstrate genetic differences, differences that are at least partially based on geographic distance, but that the isolation is either too recent or incomplete, or both, to result in marked differences in phylogeographic structure. In comparison to the genetic pattern of a subspecies of California vole that has had its habitat limited by geologic change (Neuwald 2010), the genetic pattern seen in JRBP individuals indicates that the population has had an uninterrupted presence in the area. It will be interesting to determine whether the partitioning of vole populations around the Bay has lead to genetic subdivision detectable by nuclear microsatellites, since this pattern was not detected in the slower-evolving mitochondrial genome.

The current genetic sampling technique for phylogeographic analysis heavily undersamples existing haplotype diversity, which depends on sample size and overall genetic diversity. From this one locality, we detected 11 unique haplotypes. While some of these haplotypes indicate in situ diversification, other variants are as closely related to other JRBP individuals as they are to individuals from more distant localities. Because of the lack of overall structure in 
this species across its northern distribution, increased sampling did not alter phylogeographic conclusions; however, this may not be the case when structure is present.

In conclusion, linking population genetics and phylogeography has the potential to elucidate important components of a species' ecology and evolutionary history (e.g., genetic response of populations to perturbations such as climate change). By combining fine-scale genetic analyses with longer-term phylogeographic signals in the California vole, we are able to show that gene flow is consistently high across multiple spatial scales. Despite potential impediments to gene flow, such as dispersal distance and the fragmentation of suitable habitat, the California vole represents a continuous population within its northern range, marked by isolation-by-distance but no vicariances. Overall, we are encouraged by what we see at JRBP: despite habitat encroachment from development, the population shows no signs of bottlenecking or reduced genetic diversity, and the population remains continuous within the JRBP borders and, at least in the past, remained connected to other populations throughout its northern range. Disturbances to the favored grassland habitat at JRBP are both natural-in the form of habitat diversity creating a potential barrier to migration-and anthropogenic - in the form of conversion of native grassland into one dominated by invasive grasses. Yet the rodents apparently move across both grassland types and through unfavorable habitats. The demographic and behavioral characteristics of this specieshigh philopatry and short-distance dispersalcreate a moderate but distinct pattern of increasing genetic difference with increasing geographic distance, but without sharp boundaries leading to delimited populations, both on a local and regional scale.

\section{ACKNOWLEDGMENTS}

We are grateful to Jennifer Neuwald and Chris Conroy for valuable discussion and assistance, to Nona Chiariello for providing research support, to Hillary Young for spearheading the small-mammal survey, and to Trevor Hebert for assistance with maps and GIS software. We thank Yvonne Chan, Jessica Blois, Sarah McMenamin, and an anonymous reviewer for helpful comments on previous versions of the manuscript. Funding was provided by a grant to RIA from the Andrew W. Mellon Foundation through Jasper Ridge Biological Preserve and by NSF grant \#EAR-0719429 to EAH.

\section{Literature Cited}

aars, J., J.F. Dallas, S.B. Piertney, F. Marshall, J.L. Gow, S. Telfer, and X. Lambin. 2006. Widespread gene flow and high genetic variability in populations of water voles Arvicola terrestris in patchy habitats. Molecular Ecology 15:1455-1466.

Andreassen, H.P., AND R.A. Ims. 2001. Dispersal in patchy vole populations: role of patch configuration, density dependence, and demography. Ecology 82: 2911-2926.

Atwater, B.F. 1979. Ancient processes at the site of Southern San Francisco Bay: movement of the crust and changes in sea level. Pages 31-45 in T.J. Conomos, editor, San Francisco Bay: the urbanized estuary. Pacific Division/American Association for the Advancement of Science, San Francisco, CA.

Berthier, K., N. Charbonnel, M. Galan, Y. Chaval, and J.F. Cosson. 2006. Migration and recovery of the genetic diversity during the increasing density phase in cyclic vole populations. Molecular Ecology 15: 2665-2676.

Berthier, K., M. Galan, J.C. Foltete, N. Charbonnel, AND J.F. Cosson. 2005. Genetic structure of the cyclic fossorial water vole (Arvicola terrestris): landscape and demographic influences. Molecular Ecology 14:2861-2871.

Bowen, B.S. 1982. Temporal dynamics of microgeographic structure of genetic variation in Microtus californicus. Journal of Mammalogy 63:625-638.

Broquet, T., C.A. Johnson, E. Petit, I. Thompson, F. Burel, AND J.M. FryXelL. 2006. Dispersal and genetic structure in the American marten, Martes americana. Molecular Ecology 15:1689-1697.

Clement, M., D. Posada, and K.A. Crandall. 2000. TCS: a computer program to estimate gene genealogies. Molecular Ecology 9:1657-1659.

Coltman, D.W., W.D. Bowen, and J.M. Wright. 1998. Birth weight and neonatal survival of harbour seal pups are positively correlated with genetic variation measured by microsatellites. Proceedings of the Royal Society of London Series B, Biological Sciences 265: 803-809.

ColwelL, R.K. 2006. EstimateS: statistical estimation of species richness and shared species from samples. Version 8.0 user's guide and application. Available from: http://viceroy.eeb.uconn.edu/EstimateS

ColwelL, R.K., C.X. MAO, AND J. Chang. 2004. Interpolating, extrapolating, and comparing incidence-based species accumulation curves. Ecology 85:2717-2727.

Conroy, C.J., And J.L. Neuwald. 2008. Phylogeographic study of the California vole, Microtus californicus. Journal of Mammalogy 89:755-767.

Cornuet, J.M., and G. LuiKart. 1996. Description and power analysis of two tests for detecting recent population bottlenecks from allele frequency data. Genetics 144:2001-2014.

Delport, W., J.W. Ferguson, and P. Bloomer. 2005. Characterization of six microsatellite loci in the African wild silk moth (Gonometa postica, Lasiocampidae). Molecular Ecology Notes 5:860-862. 
Evanno, G., S. Regnaut, and J. Goudet. 2005. Detecting the number of clusters of individuals using the software STRUCTURE: a simulation study. Molecular Ecology 14:2611-2620.

Excoffier, L., J. Novembre, and S. Schneider. 2000. SIMCOAL: a general coalescent program for the simulation of molecular data in interconnected populations with arbitrary demography. Journal of Heredity 91:506-509.

Falush, D., M. Stephens, and J.K. Pritchard. 2003. Inference of population structure using multilocus genotype data: linked loci and correlated allele frequencies. Genetics 164:1567-1587.

Frankham, R., J.D. Ballou, and D.A. Briscoe. 2002. Introduction to conservation genetics. Cambridge University Press, Cambridge.

Frantz, A.C., S. Cellina, A. Krier, L. Schley, and T. BURKE. 2009. Using spatial Bayesian methods to determine the genetic structure of a continuously distributed population: clusters or isolation by distance? Journal of Applied Ecology 46:493-505.

Futurma, D.J. 1998. Evolutionary biology. 3rd edition. Sinauer Associates, Sunderland, MA.

Gauffre, B., A. Estoup, V. Bretagnolle, and J.F. Cosson. 2008. Spatial genetic structure of a small rodent in a heterogeneous landscape. Molecular Ecology 17:46194629 .

GILL, A.E. 1980. Partial reproductive isolation of subspecies of the California vole Microtus californicus. Pages 105-118 in N.N. Vorontsov and J.M. Van Brink, editors, Animal genetics and evolution: selected papers of the 14th International Congress of Genetics. BV Publishers: The Hague, Netherlands; Moscow, USSR. 21-30 August 1978.

Giusti, G.A. 1985. Home ranges, activity patterns and relative densities of meadow mice (Microtus californicus) and harvest mouse (Reithrodontomys megalotis) on Jasper Ridge Biological Preserve. Master's thesis, San Francisco State University, San Francisco, CA.

Gotelli, N.J., AND R.K. Colwell. 2001. Quantifying biodiversity: procedures and pitfalls in the measurement and comparison of species richness. Ecology Letters 4:379-391.

Greenwald, G.S. 1957. Reproduction in a coastal California population of the field mouse, Microtus californicus. University of California Publications in Zoology 54:421-446.

Guillot, G., F. Mortier, And A. Estoup. 2005. GENELAND: a computer package for landscape genetics. Molecular Ecology Notes 5:712-715.

Guillot, G., F. SAntos, And A. Estoup. 2008. Analysing georeferenced population genetics data with Geneland: a new algorithm to deal with null alleles and a friendly graphical user interface. Bioinformatics 24 : 1406-1407.

Hadly, E.A., U. Ramakrishnan, Y.L. Chan, M. van Tuinen, K. O’Keefe, P.A. Spaeth, and C.J. Conroy. 2004 Genetic response to climatic change: insights from ancient DNA and phylochronology. PLoS Biology 2: 1600-1609.

HaLl, E.R. 1981. The mammals of North America. 2nd edition. Wiley, New York, NY.

HaRDY, O.J., AND X. VEKEMANS. 2002. SPAGeDi: a versatile computer program to analyse spatial genetic structure at the individual or population levels. Molecular Ecology Notes 2:618-620.
Hartl, D.L., And A.G. Clark. 1997. Principles of population genetics. 3rd edition. Sinauer Associates, Sunderland, MA.

Heske, E.J. 1987. Spatial structuring and dispersal in a high density population of the California vole Microtus californicus. Holarctic Ecology 10:137-148.

Hobbs, R.J., S. Yates, And H.A. MoONEY. 1997. Long-term data reveal complex dynamics in grassland in relation to climate and disturbance. Ecological Monographs 77:545-568.

Hooper, E.T. 1944. San Francisco Bay as a factor influencing speciation in rodents. University of Michigan Press, Ann Arbor, MI.

Ishibashi, Y., Y. YoshinaGa, T. SAITOH, S. ABE, H. IIDA, AND M.C. YoshIDA. 1999. Polymorphic microsatellite DNA markers in the field vole Microtus montebelli. Molecular Ecology 8:163-164.

KeLloGG, R. 1918. A revision of the Microtus californicus group of meadow mice. University of California Publications in Zoology 21:1-42.

Krebs, C.J. 1966. Demographic changes in fluctuating populations of Microtus californicus. Ecological Monographs 36:239-273.

LAVERGNe, S., AND J. Molofsky. 2007. Increased genetic variation and evolutionary potential drive the success of an invasive grass. Proceedings of the National Academy of Sciences of the United States of America 104:3883-3888.

LIDICKER, W.Z. 1973. Regulation of numbers in an island population of the California vole: a problem in community dynamics. Ecological Monographs 43:271302.

Lowe, A., S. Harris, And P. Ashton. 2004. Ecological genetics: design, analysis, and application. Blackwell Science, Oxford

Mayer, C., K. Schiegg, and G. Pasinelli. 2009. Patchy population structure in a short-distance migrant: evidence from genetic and demographic data. Molecular Ecology 18:2353-2364.

NEI, M. 1987. Molecular evolutionary genetics. Columbia University Press, New York, NY.

NeuwaLD, J.L. 2010. Population isolation exacerbates conservation genetic concerns in the endangered Armagosa vole, Microtus californicus scirpensis. Biological Conservation 143:2028-2038.

Peakall, R., and P.E. Smouse. 2006. GENALEX 6: genetic analysis in Excel. Population genetic software for teaching and research. Molecular Ecology Notes 6:288-295.

Piry, S., G. Luikart, and J.M. Cornuet. 1999. BOTTLENECK: a computer program for detecting recent reductions in the effective population size using allele frequency data. Journal of Heredity 90:502-503.

Pritchard, J.K., M. Stephens, and P. Donnelly. 2000. Inference of population structure using multilocus genotype data. Genetics 155:945-959.

Pritchard, J.K., X. Wen, and D. Falush. 2007. Documentation for structure software. Version 2.2. Available from: http://pritch.bsd.uchicago.edu/structure.html

RITLAND, K. 1996. Estimators for pairwise relatedness and individual inbreeding coefficients. Genetical Research 67:175-185.

Rousset, F. 1997. Genetic differentiation and estimates of gene flow from $F$-statistics under isolation by distance. Genetics 145:1219-1228.

2000. Genetic differentiation between individuals. Journal of Evolutionary Biology 13:58-62. 
2008. GENEPOP'007: a complete re-implementation of the GENEPOP software for Windows and Linux. Molecular Ecology Resources 8:103-106.

SALVIONI, M., AND W.Z. LIDICKER. 1995. Social organization and space use in California voles: seasonal, sexual, and age-specific strategies. Oecologia 101:426-438.

Smith, M.F., AND J.L. PATTON. 1993. The diversification of South American murid rodents: evidence from mitochondrial DNA sequence data for the akodontine tribe. Biological Journal of the Linnean Society 50:149177.

Smouse, P.E., R. Peakall, and E. Gonzales. 2008. A heterogeneity test for fine-scale genetic structure. Molecular Ecology 17:3389-3400.

SoulE, M.E. 1980. Thresholds for survival: maintaining fitness and evolutionary potential. Pages 151-170 in M.E. Soule and B.A. Wilcox, editors, Conservation biology: an evolutionary-ecological perspective. Sinauer Associates, Sunderland, MA.

Spaeth, P.A., M. van Tuinen, Y.L. Chan, D. Terca, and E.A. HADLY. 2009. The phylogeography of the longtailed vole, Microtus longicaudus, in the tectonically and glacially dynamic landscape of the Central Rocky Mountains. Journal of Mammalogy 90:571-584.

Stewart, W.A., S.B. Piertney, F. Marshall, X. Lambin, AND S. TELFER. 1999. Metapopulation genetic structure in the water vole, Arvicola terrestris, in NE Scotland. Biological Journal of the Linnean Society 68: 159-171.

SWOFFORD, D.L. 2003. PAUP*: phylogenetic analysis using parsimony (*and other methods). Version 4. Sinauer Associates, Sunderland, MA.

Van de Zande, L., R.C. Van Apeldoorn, A.F. BlijdenStein, D. De Jong, W. Van Delden, and R. Bijlsma. 2000. Microsatellite analysis of population structure and genetic differentiation within and between populations of the root vole, Microtus oeconomus, in the Netherlands. Molecular Ecology 9:1651-1656.

Waser, P.M., AND L.F. ElliotT. 1991. Dispersal and genetic structure in kangaroo rats. Evolution 45:935943.

Received 27 August 2009 Accepted 19 February 2010 